\title{
Anastomose mamária interna-artéria pulmonar para o tratamento paliativo das cardiopatias congênitas cianóticas
}

\author{
João J. CARNEIRO*, Walter V. A. VICENTE*, Olair A. QUEIROZ*, João M. TANNÚS FILHO**, José
} A. MARIN NETO*, João A. GRANZOTTI, Albert A. SADER*

RBCCV $44205-110$

CARNEIRO, J. J.; VICENTE, W. V. A.; QUEIROZ, O. A.; TANNÚS FILHO, J. M.; MARIN NETO, J. A.; GRANZOTTI, J. A.; SADER, A. A. - Anastomose mamária interna-artéria pulmonar para o tratamento paliativo das cardiopatias congênitas cianóticas. Rev. Bras. Cir. Cardiovasc., 5(2): 79-85, 1990.

RESUMO: As anastomoses sistêmico-pulmonares continuam sendo um importante procedimento no tratamento e na preparação dos cardiopatas cianóticos, com hipoplasia das artérias pulmonares, para a correção total. Todas têm vantagens e desvantagens e os resultados dependerão de idade e peso dos pacientes e da complexidade da cardiopatia. O presente estudo relata a experiência com sete casos de anastomoses mamária-artéria pulmonar, realizadas através de toracotomia direita (5) e esquerda (2), em pacientes portadores de tetralogia de Fallot (quatro femininos e três masculinos). A idade variou de dois a 63 meses $(m=18,4)$, com peso médio de $7,9 \mathrm{~kg}$. Seis apresentavam graus variados de hipoplasia pulmonar. Houve dois óbitos pós-operatórios (1: e $2^{\circ}$. dias), devidos a trombose da artéria mamária, no local de seu clampleamento. Dois pacientes foram submetidos a correção total (um mês e três anos após): no 1: (menina de 24 meses, $10 \mathrm{~kg}$ de peso), a anastomose foi feita pelas más condiçōes gerais, apesar do bom tamanho das pulmonares. No 2 : (menino de 15 meses, $8,1 \mathrm{~kg}$, no qual uma operação de BlalockTaussig clássica foi feita e trombosou no 3 : mês de vida), foi possível observar o progressivo desenvolvimento das artérias pulmonares, por estudo hemodinâmico. Em ambos, o funcionamento da anastomose era perfeito e a correção foi bem sucedida. Três outros pacientes aguardam o momento oportuno para a correção final. Apesar do reduzido número e do curto tempo de observação, acredita-se que a anastomose mamáriapulmonar possa ser outra opção paliativa para os portadores de cardiopatias congênitas cianogênicas, com hipoplasia das artérias pulmonares e que os melhores resultados devam ser obtidos nos pacientes maiores, fora de situaçōes de emergência e com pequenas artérias pulmonares.

DESCRITORES: artéria mamária interna-artéria pulmonar, anastomose.

\section{INTRODUÇÃO}

O tratamento paliativo das cardiopatias cianogênicas com hipofluxo pulmonar tem, ainda hoje, indicação destacada para os casos em que a correção deva ser feita mais tardiamente, ou que a anatomia complexa impede a cirurgia corretiva final.

A técnica descrita por BLALOCK \& TAUSSIG ${ }^{4}$, em 1945, continua sendo empregada, em suas formas clás-

Trabalho realizado no Hospital das Clinicas da Faculdade de Medicina de Ribeirão Preto. Disciplina de Cirurgia Torácica e Cardiovascular. Ribeirão Preto, SP, Brasil.

Apresentado ao 17: Congresso Nacional de Cirurgia Cardiaca. Belo Horizonte, MG, 6 e 7 de abril, 1990.

* da Faculdade de Medicina de Ribeirâo Preto - U.S.P

** da Faculdade de Medicina da Universidade Federal de Uberlândia.

Endereço para separatas: Joăo J. Carneiro. Faculdade de Medicina de Ribeirão Preto. Departamento de Cirurgia, Ortopedia e Traumatologia. 14028 Ribeirâo Preto, SP, Brasil. 
CARNEIRO, J. J.; VICENTE, W. V. A.; QUEIROZ, O. A.; TANNÚS FILHO, J. M.; MARIN NETO, J. A.; GRANZOTTI, J. A.; SADER, A. A. - Anastomose mamária interna-artéria pulmonar para o tratamento paliativo das cardiopatias congênitas cianóticas.

Rev. Bras. Cir. Cardiovasc., 5(2): 79-85, 1990.

sica ou modificada $2,5,11,12,15$. Os resultados obtidos variam de acordo com os grupos etários e morfológicos e, principalmente, com o tamanho dos pacientes.

Novas opções foram criadas com as técnicas descritas por POTTS et alii ${ }^{21}$, WATERSTON ${ }^{29}$ e GAZZANIGA et alii " , porém todas têm vantagens e desvantagens inerentes à sua realizaçāo ou ao seu fechamento. Estas consideraçōes ficam mais críticas quando as artérias pulmonares são de calibre muito reduzido.

Com base neste fato, no uso freqüente das artérias mamárias para a revascularização miocárdica e na observaçāo das fístulas artério-venosas periféricas para hemodiálise, teve origem a possibilidade de uma outra opçāo paliativa para as cardiopatias congênitas cianogênicas, com hipofluxo pulmonar: a anastomose mamáriapulmonar. Esta cirurgia já havia sido realizada por alguns autores em condições especiais ${ }^{6,17,22}$.

As fístulas artério-venosas periféricas, após sua maturação, são capazes de manter fluxo sangüíneo suficiente para hemodiálise e promovem o desenvolvimento acentuado das veias de drenagem. Ambos são resultados desejados para as anastomoses sistêmico-pulmonares.

No Hospital das Clínicas da Faculdade de Medicina de Ribeirão Preto (USP), a partir de 1986, sete pacientes foram submetidos a anastomose mamária interna-pulmonar. O presente trabalho relata a experiência inicial destes casos.

\section{CASUÍSTICA E MÉTODOS}

O grupo é constituído de sete crianças (três masculinos e quatro femininos), com idade de dois a 63 meses ( $m=18,4 \mathrm{~m}$ ), todas portadoras de tetralogia de Fallot com crises hipoxêmicas e, seis delas, com hipoplasia pulmonar, constatada por estudo hemodinâmico. Em um caso (menina de 2 anos de idade, $10 \mathrm{~kg}$ de peso), as pulmonares eram de bom tamanho, porém, as condiçōes gerais da paciente contra-indicaram a correção total. Um menino de 15 meses apresentava anastomose clássica de Blalock-Taussig, realizada e trombosada no 3 : mês de vida. A paciente de mais idade (63 meses, 13,8 kg), além de hipoplasia, apresentava assimetria das artérias pulmonares, sendo a direita de calibre extremamente reduzido. A Tabela 1 resume os dados referentes ao grupo.

Todos os pacientes foram operados sob anestesia geral e respiraçāo controlada (thionembutal, morfina ou fentanil, fluothane e pancurinium). Naqueles que apresentavam micro-hematócrito acima de $50 \%$, realizou-se hemodiluição até à obtenção de valores ao redor de $45 \%, \operatorname{logo}$ após a indução anestésica e utilizando-se sangria e plasma fresco. Heparinização sistêmica (1 mg/ $\mathrm{kg}$ de peso) foi empregada em todos os pacientes, sem a correspondente neutralização com protamina.
TABELA 1

RESUMO DOS PACIENTES OPERADOS

\begin{tabular}{lcrccrl}
\hline Caso Sexo & $\begin{array}{c}\text { Peso } \\
(\mathrm{kg})\end{array}$ & $\begin{array}{c}\text { Sup. corp. } \\
\left(\mathrm{m}^{2}\right)\end{array}$ & $\begin{array}{c}\text { Ht } \\
\%\end{array}$ & $\begin{array}{c}\text { Idade } \\
\text { (meses) }\end{array}$ & \multicolumn{1}{c}{$\begin{array}{c}\text { Lesóes } \\
\text { associadas }\end{array}$} \\
\hline 1 & $\mathrm{~F}$ & 7,18 & 0,37 & 60 & 9 & C.I.A. \\
2 & $\mathrm{M}$ & 8,10 & 0,42 & 47 & 15 & B. T. trombosado \\
3 & $\mathrm{M}$ & 5,73 & 0,28 & 40 & 7 & D.V.S.V.D. \\
$4^{*}$ & $\mathrm{~F}$ & 10,00 & 0,49 & 57 & 24 & - \\
5 & $\mathrm{M}$ & 8,10 & 0,42 & 40 & 9 & - \\
6 & $\mathrm{~F}$ & 2,55 & 0,18 & 40 & 2 & - \\
7 & $\mathrm{~F}$ & 13,80 & 0,62 & 65 & 63 & Hip. pulm. assim. \\
\hline Média & & 7,90 & 0,39 & 49,8 & 18,4 & \\
\hline
\end{tabular}

- paciente com bons ramos pulmonares; $\mathrm{Ht}=$ microhematócrito; C.I.A. = comunicaçāo interatrial; B. T. Trombosado = Blalock-Taussig trombosado; D.V.S.V.D. = dupla via de saida do ventrículo direito; HIP. PULM. ASSIM. = hipoplasia assimética das artérias pulmonares.

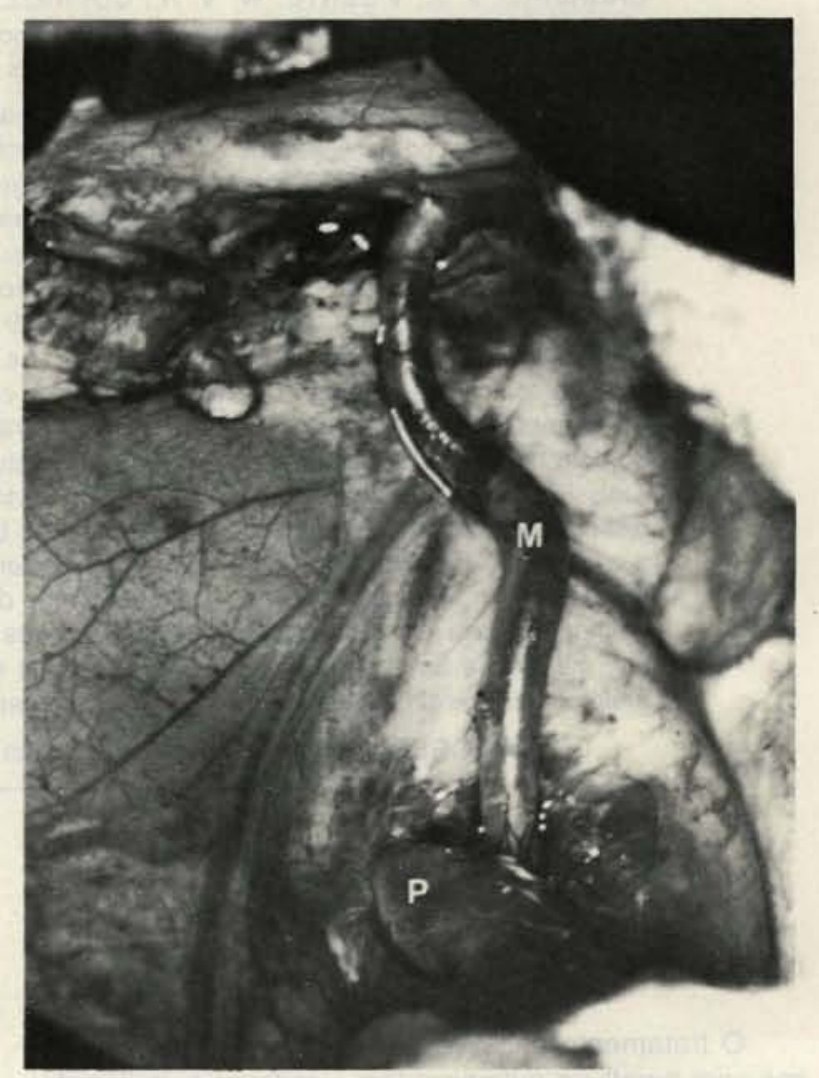

Fig. 1 - Anastomose mamária-pulmonar esquerda (caso 2). $\mathrm{M}=$ artéria mamária interna $\mathrm{P}=$ artéria pulmonar

Foram feitas cinco toracotomias à direita e duas à esquerda. A artéria mamária foi anastomosada terminalmente às artérias pulmonares, direita ou esquerda, utilizando-se Prolene 7-0. Nesses casos, $50 \%$ da anastomose foi realizada com pontos separados (Figura 1). 
CARNEIRO, J. J.; VICENTE, W. V. A.; QUEIROZ, O. A.; TANNÚS FILHO, J. M.; MARIN NETO, J. A.; GRANZOTTI, J. A.; SADER, A. A. - Anastomose mamária interna-artéria pulmonar para o tratamento paliativo das cardiopatias congênitas cianóticas. Rev. Bras. Cir. Cardiovasc., 5(2): 79-85, 1990.

Em três casos, a sutura foi contínua, utilizando-se PDS* 7-0 (Polydioxanone) Suture - Ethicon.

Os controles gasométricos foram feitos em nível capilar, uma vez que, considerando-se os procedimentos fechados, não se cateterizou artéria para monitorização.

\section{RESULTADOS}

Não houve óbitos no intra-operatório. Dois pacientes (dois e sete meses) faleceram no pós-operatório imediato ( 1 : e 2 . dias), por trombose no local do clampeamento da artéria mamária interna.

Dois pacientes foram submetidos a correção total. Uma menina de 24 meses em que as artérias pulmonarres eram de bom calibre, mas que suas condiçōes gerais contra-indicaram a correçāo total, foi operada um mês após a anastomose mamária-pulmonar. O segundo paciente, com o Blalock-Taussig trombosado à direita e a anastomose mamária-pulmonar realizada à esquerda, foi reestudado após 18 e 16 meses (Fig. 2). Nesta última oportunidade, foi submetido a correção total com ligadura da mamária. Em ambos os casos, as anasto-
TABELA 2

RESULTADOS E EVOLUÇĀO PÓS-OPERATÓRIA

\begin{tabular}{|c|c|c|c|c|c|c|}
\hline Caso & Lado & $\begin{array}{c}\text { Sat. } \\
\text { pré } \\
\%\end{array}$ & $\begin{array}{c}\text { Sat. } \\
\text { poss } \\
\%\end{array}$ & $\begin{array}{c}\text { Tempo } \\
\text { (meses) }\end{array}$ & $\begin{array}{c}\text { P.O. } \\
\text { imediato }\end{array}$ & $\begin{array}{l}\text { P.O. } \\
\text { tardio }\end{array}$ \\
\hline 1 & D & 33 & 60 & 44 & Boa & Aguarda correçāo \\
\hline 2 & E & 31 & 77 & 36 & Boa & Correção total \\
\hline 3 & $E$ & 29 & 60 & - & Óbito & - \\
\hline 4 & D & 36 & 75 & 1 & Boa & Correção total \\
\hline 5 & D & 32 & 68 & 15 & Boa & Aguarda correção \\
\hline 6 & D & 32 & 58 & - & Óbito & - \\
\hline 7 & D & 29 & 76 & 3 & Boa & Aguarda correção \\
\hline
\end{tabular}

Média $31,7 \quad 67,7 \quad 19,8$

moses estavam pérvias, a ligadura da mamária foi fácil e a correçāo total, bem sucedida.

Os três pacientes restantes encontram-se em seguimento clínico ( 4 anos, 15 e 4 meses), aguardando a melhor oportunidade para reestudo e correçăo total.

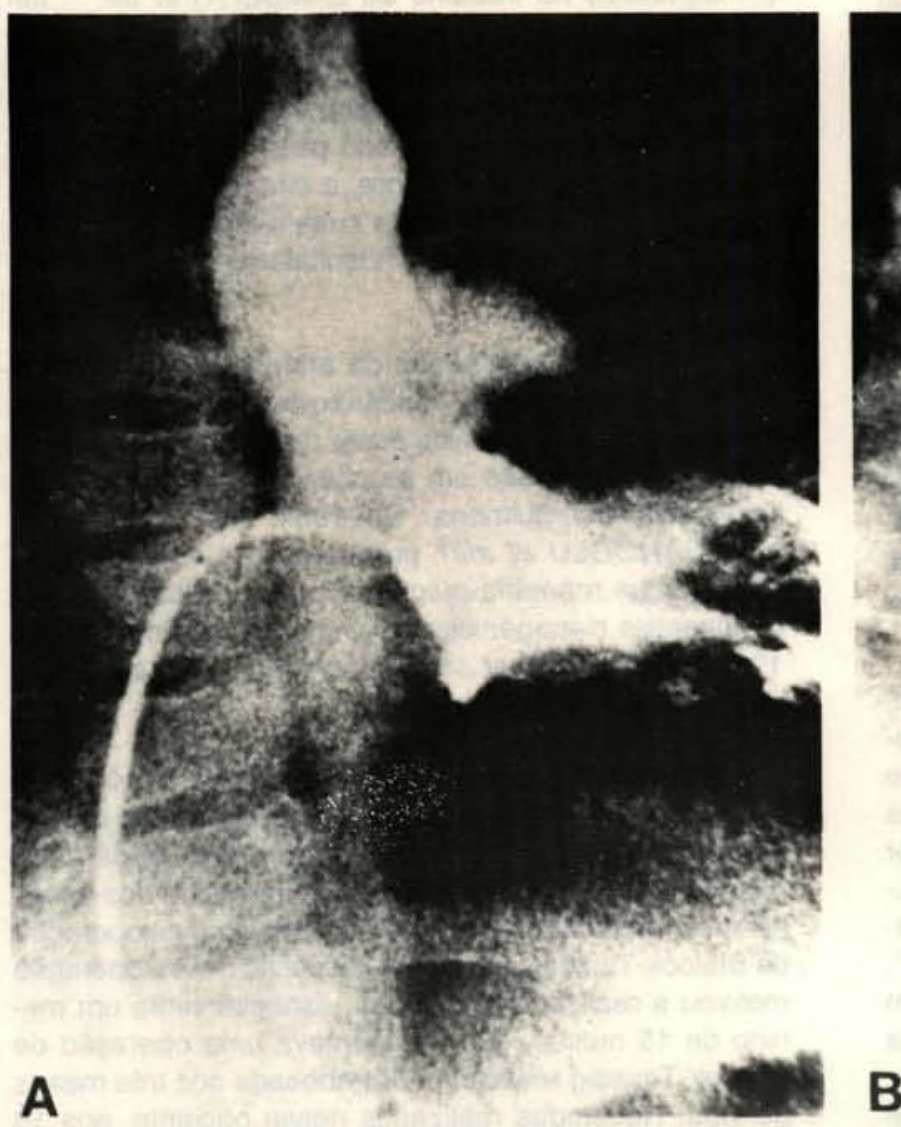

Fig. 2-Caso 2. A = Blalock à direita trombosado. $\mathrm{B}=$ Aortografia 18 meses após anastomose mamária-pulmonar à esquerda. 
CARNEIRO, J. J.; VICENTE, W. V. A.; QUEIROZ, O. A.; TANNÚS FILHO, J. M.; MARIN NETO, J. A.; GRANZOTTI, J. A.; SADER, A. A. - Anastomose mamária interna-artéria pulmonar para o tratamento paliativo das cardiopatias congênitas cianóticas.

Rev. Bras. Cir. Cardiovasc., 5(2): 79-85, 1990.

A saturação média do sangue capilar por oxigênio, no pré-operatório foi de $31,7 \%$, elevando-se para $67,7 \%$ no pós-operatório imediato.

A Tabela 2 resume os resultados e a evolução do grupo. No paciente submetido a reestudo visando à correção total três anos após, notou-se que a saturação do sangue arterial por oxigênio, pré-operatório, de 58,9\% (em $\mathrm{O}_{2}$ puro) passou para $89 \%$ (em ar atmosférico).

\section{DISCUSSĀO}

As cirurgias paliativas, iniciadas com o pioneirismo de BLALOCK \& TAUSSIG ${ }^{4}$, em 1945 , continuam tendo seu papel de destaque no tratamento e preparação dos cardiopatas congênitos cianóticos, com hipofluxo pulmonar $1,2,4,11,14,21,28,29$.

Mas as restrições à operação de Blalock-Taussig clássica são, de há muito, conhecidas: tamanho e diâmetro da artéria subclávia; acotovelamento em sua origem; repuxamento da artéria pulmonar e seqüelas isquêmicas no membro superior correspondente. Nesse sentido, MEARNS et alii ${ }^{18}$, em 1978, relataram um caso no qual a grangrena do membro foi evitada por um enxerto venoso carótida-axilar, utilizando segmento da safena interna. Por outro lado, estudos realizados por SKROVÁNEK et $a{ }^{2} i^{23}$ demonstram que o membro que perde a artéria subclávia comporta-se, do ponto de vista do fluxo sangüineo, como possuindo uma obstrução arterial crônica. Segundo EDMUNDS et alii ${ }^{10}$, para que se obtenha uma boa anastomose subclávia-pulmonar, o diâmetro ideal da primeira artéria deva ser de $2,5 \mathrm{~mm}$. As anastomoses realizadas com artérias menores que $2,3 \mathrm{~mm}$ trombosaram.

Em 1946, POTTS et alii ${ }^{21}$ descreveram a anastomose da aorta descendente com a artéria pulmonar, com a qual pretendiam ter resolvido o problema do diâmetro da fistula. Entretanto, năo foram estes os resultados obtidos e, além da freqüente doença vascular pulmonar desenvolvida, a dificuldade técnica para sua ligadura, no momento da correção final, tornou-a pouco recomendável.

WATERSTON ${ }^{28}$, em 1962 descreveu a anastomose da aorta ascendente com a artéria pulmonar direita, intrapericárdica, a qual, exigindo menor dissecçāo que a de Blalock, parecia resolver o problema, principalmente nas crianças menores. Esse alento tornou-se ainda maior com as modificaçōes introduzidas por COOLEY \& HALLMAN $^{7}$. Na realidade, esta técnica tem inúmeras vantagens sobre a de Blalock-Taussig 2, 3, 9, 20, 24-27, 29, 30, porém várias desvantagens foram sendo apontadas com o passar do tempo: falhas no funcionamento da fístula (de 3 a 14\%), insuficiência cardiaca congestiva, acotovelamento da artéria pulmonar direita, fluxo preferencial para a direita, com falha no desenvolvimento do tronco pulmonar. Quando da correção final, cuidados especiais precisam ser tomados para não persistir a estenose da artéria pulmonar direita $^{9,25,30}$. Apesar disso, pela relativa facilidade de execução e possibilidade de sua associaçāo com outras intervençōes, foi a anastomose preferida durante muito tempo ${ }^{2,9}$.

A introdução do politetrafluoretileno (PTFE) como prótese venosa, por Soyers, em 1972, abriu um novo campo na confecção das fístulas sistêmico-pulmonares. Foram GAZZANIGA et alii ${ }^{11}$ que, em 1976, propuseram a interposição de um enxerto de PTFE entre a aorta e a pulmonar. Se o novo material permitia a realização dessas fístulas com maior facilidade que a operaçāo de Blalock-Taussig original em crianças menores, ele não crescia com a criança, a qual, após alguns anos, precisava se submeter a outra cirurgia. Usando-se tubos de diâmetros maiores, os pacientes desenvolviam insuficiência cardiaca congestiva, com evolução insatisfatória $^{8}$. Foi do estudo desses enxertos que KUSUHARA et alii ${ }^{13}$ demonstraram que o valor ideal de fluxo através deles era de 1,6 a 2,41/min/m². Em 1979, LAMBERTI et alii ${ }^{15}$ comprovaram a importância desses fatos.

Em 1984, ILBAWI et alii ${ }^{12}$ e LAMBERTI et alii ${ }^{16}$ publicaram os resultados do que passou a ser conhecido como Blalock-Taussig modificado e proposto por Stark, em discussão no trabalho de LAMBERTI et alii ${ }^{15}$ : um tubo de PTFE de $5 \mathrm{~mm}$ ou $6 \mathrm{~mm}$ de diâmetro, independentemente do calibre da artéria subclávia, é utilizado para confecção da fístula sistêmico-pulmonar. O fluxo, através do enxerto, é regulado pelo calibre da artéria. Como o tubo tem bom calibre, a criança pode crescer, que ele continua adaptado a suas necessidades. Com essa técnica, a mortalidade hospitalar e a trombose são de baixa incidência.

A utilização continuada da artéria mamária interna na revascularização do miocárdio com bons resultados e o aprimoramento técnico desta cirurgia são os responsáveis pelo interesse em se criar uma nova opção de fistula sistêmico-pulmonar. Em 1984, SIEVERS et alii ${ }^{22}$ e COBANOGLU et alii $^{6}$ propuseram a realização da anastomose mamária-pulmonar para o tratamento das cardiopatias cianogênicas com hipoplasia pulmonar. Em 1989, LONGAKER et alii ${ }^{17}$ descreveram um caso operado com sucesso.

No Hospital das Clínicas da Faculdade de Medicina de Ribeirão Preto (USP), a primeira anastomose mamária interna-pulmonar foi realizada em 1986, dada a impossibilidade, pela presença de malformaçāo dos vasos da base, de se utilizar a artéria subclávia para a operaçāo de Blalock-Taussig clássico. O sucesso dessa operação motivou a realização de outras, especialmente um menino de 15 meses, que apresentava uma operação de Blalock-Taussig realizada e trombosada aos três meses de vida. Reestudos realizados nesse paciente, aos 18 e 36 meses do pós-operatório, demonstraram, não so- 
CARNEIRO, J. J.; VICENTE, W. V. A.; QUEIROZ, O. A.; TANNÚS FILHO, J. M.; MARIN NETO, J. A.; GRANZOTTI, J. A.; SADER, A. A. - Anastomose mamária interna-artéria pulmonar para o tratamento paliativo das cardiopatias congênitas cianóticas.

Rev. Bras. Cir. Cardiovasc., 5(2): 79-85, 1990.

mente o enxerto patente, como também o crescimento da artéria pulmonar e seus ramos. A melhora gasométrica nāo deixava dúvidas quanto ao seu funcionamento. A cirurgia corretiva total foi realizada nessa época e a ligadura da artéria mamária, aumentada de calibre, não ofereceu qualquer dificuldade. Tivemos dois óbitos pósoperatórios, causados por trombose da artéria mamária, no local de clampeamento com a anastomose pérvia, em ambos os casos. Isto se deveu ao uso de instrumental vascular grosseiro e inadequado para a artéria mamária interna.

Dos três pacientes que aguardam o momento oportuno para reconstrução, o último deles foi o que nos causou melhor impressão; a anastomose foi feita na arté- ria pulmonar direita, extremamente hipoplásica, com bom resultado.

Não podemos esquecer que as anastomoses são de pequeno diâmetro e que, para funcionarem plenamente, necessitam de adaptação e, até mesmo, de suporte farmacológico, nos primeiros dias.

Apesar do curto tempo de observação e do reduzido número de casos, acredita-se que a anastomose internapulmonar pode ser mais uma opção paliativa para as cardiopatias cianogênicas com hipofluxo pulmonar, tendo, como melhores indicaçōes, as crianças maiores, fora de crises hipoxêmicas graves e com artérias pulmonares de reduzido diâmetro. Para condiçōes de emergência, a operação de Blalock-Taussig modificada ${ }^{12,16}$, ou mesmo a fístula central ${ }^{2}$ poderão ser empregadas.

RBCCV 44205-110

CARNEIRO, J. J.; VICENTE, W. V. A.; QUEIROZ, O. A.; TANNÚS FILHO, J. M.; MARIN NETO, J. A.; GRANZOTTI, J. A.; SADER, A. A. - Internal mammary-pulmonary artery anastomosis for cyanotic congenital heart disease. Rev. Bras. Cir. Cardiovasc., 5(2): 79-85, 1990.

ABSTRACT: The systemic-pulmonary shunts are an important procedure to treat and prepare, for the final correction, cyanotic patients with hypoplastic pulmonary arteries. All of them have advantages and disadvantages, and the results will depend on the age and weight of the patients and the complexity of their heart disease. The present study reports seven internal mammary-pulmonary anastomosis, made through right (2) or left (5) thoracotomies, in patients with Tetralogy of Fallot and hypoplastic pulmonary arteries, from 2 to 63 months of age $(m=18,4)$ and mean weight of $7.9 \mathrm{~kg}$. There were two deaths $\left(1^{\text {st }}\right.$ and $2^{\text {nd }}$ days), due to thrombosis of the internal mammary, where it was clamped. Two patients went to total correction (1 month and 3 years later). In the first case, the shunt was made due to the general conditions of the child, despite the good size of the pulmonary arteries. In the second case (a 15 months old boy, in whom a classical Blalock-Taussig shunt was made and thrombosed in the $3^{\text {rd }}$ month of life) was possible to follow the progressive enlargement of the pulmonary arteries. In both the shunts were well functioning and the total correction was successful. Three patients are waiting for their best time for final procedures. Despite the small number and the short period of observations, the authors do believe that the internal mammary-pulmonary artery anastomisis is palliative option for these patients. The best results are obtained among the eldest children that are not in an emergency situation and not involving small pulmonary arteries.

DESCRIPTORS: internal mammary artery-pulmonary artery anastomosis.

\section{REFERÊNCIAS BIBLIOGRÁFICAS}

1 ARCINIEGAS, E.; FAROOKI, Z. Q.; HAKIMI, M.; PERRY, B. L.; GREEN, E. W. - Classic shunting operations for congenital cyanotic heart defects. J. Thorac. Cardiovasc. Surg., 84: 88-96, 1982.

2 BARRAGRY, T. P.; RING, S. W.; BLATCHFORD, J. W.; FOKER, J. E. - Central aorta-pulmonary artery shunts in neonates with complex cyanotic congenital heart disease. J. Thorac. Cardiovasc. Surg., 93: 767-774, 1987.

3 BERNHARD, W. F.; JONES, J. E.; FRIEDBERG, D. Z.; LITWIN, S. B. - Ascending aorta-right pulmonary arte- ry shunt in infants and older patients with certain types of cyanotic congenital heart disease. Circulation, 43: 580-584, 1971.

4 BLALOCK, A. \& TAUSSIG, H. B. - The surgical treatment of malformations of the heart, in which there is pulmonary stenosis or pulmonary atresia. JAMA, 128: 189-202, 1945.

5 BOVE, E. L.; SONDHEIMER, H. M.; KAVEY, R. E. W.; BYRUM, C. J.; BLACKMAN, M. S.; PARKER Jr., F. B. - Subclavian-pulmonary artery shunts with polytetrafluorethylene interposition grafts. Ann. Thorac. Surg., 37: 88-91, 1984. 
CARNEIRO, J. J.; VICENTE, W. V. A.; QUEIROZ, O. A.; TANNÚS FILHO, J. M.; MARIN NETO, J. A.; GRANZOTTI, J. A.; SADER, A. A. - Anastomose mamária interna-artéria pulmonar para o tratamento paliativo das cardiopatias congênitas cianóticas. Rev. Bras. Cir. Cardiovasc., 5(2): 79-85, 1990.

6 COBANOGLU, A.; ABBRUZZESE, P.; BRAUnER, D. O.; FERRE, B.; ISSENBERG, H.; STARR, A. - Therapeutic considerations in congenital absence of the right pulmonary artery. J. Cardiovasc. Surg., 25:241-245, 1984.

7 COOLEY, D. A. \& HALLMAN, G. L. - Intrapericardial aortic-right pulmonary arterial anastomosis. Surg. Gynec. Obstet., 122: 1084-1086, 1966.

8 DONAHOO, J. S.; GARDNER, T. J.; ZAHKA, K.; KIDD, B. S. L. - Systemic-pulmonary shuns in neonates and infants using microporous expanded polytetrafluorethylene: immediate and late results. Ann. Thorac. Surg., 30: 146-150, 1980.

9 EBERT, P. A.; GAY Jr., W. A.; OLDHAM, H. N. - Management of aorta-pulmonary artery anastomosis during total correction of tetralogy of Fallot. Surgery, 71: 231-234, 1972.

10 EDMUNDS Jr., L. H.; STEPHENSON, L. W.; GADZIK, J. P. - The Blalock-Taussig anastomosis in infants younger than 1 week of age. Circulation, 62: 597-603, 1980.

11 GAZZANIGA, A. B.; LAMBERTI, J. J.; SIEWERS, R. D.; SPERLING, D. R.; DIETRICK, W. R.; ARCILLA, R. A.; REPLOGLE, R. L. - Arterial prosthesis of microporous expanded polytetrafluorethylene for construction of aorta-pulmonary shunts. J. Thorac. Cardiovasc. Surg., 72: 357-363, 1976.

12 ILBAWI, M. N.; GRIECO, J.; DeLEON, S. Y.; IDRISS, F. S.; MUSTER, A. J.; BERRY, T. E.; KLICH, J. - Modified Blalock-Taussig shunt in newborn infants. J. Thorac. Cardiovasc. Surg., 88: 770-775, 1984.

13 KUSUHARA, K.; MIKI, S.; UEDA, Y.; OHKITA, Y.; TAHATA, T.; KOMEDA, M. - Optimal flow of aorta-pulmonary artery shunt in patients with cyanotic heart disease. Ann. Thorac. Surg., 44: 128-134, 1987.

14 LAKS, H.; FAGAN, L.; BARNER, H. B.; WILLMAN, V. L. - The Blalock-Taussig shunt in the neonate. Ann. Thorac. Surg., 25: 220-224, 1978.

15 LAMBERTI, J. J.; CAMPBELL, C.; REPLOGLE, R. L.; ANAGNOSTOPOULOS, C.; LIN, C. Y.; CHIEMMONGKOLTIP, P.; ARCILLA, R. - The prosthetic (Teflon) central aortopulmonary shunt for cyanotic infants less than three weeks old: results and long-term follow-up. Ann. Thorac. Surg., 28: 568-577, 1979.

16 LAMBERTI, J. J.; CARLISLE, J.; WALDMAN, J. D.; LODGE, F. A.; KIRKPATRICK, S. E.; GEORGE, L.; MATHEWSON, J. W.; TURNER, S. W.; PAPPELBAUM, S. J. - Systemic-pulmonary shunts in infants and children: early and late results. J. Thorac. Cardiovasc. Surg., 88: 76-81, 1984.

17 LONGAKER, M. T.; MERRICK, S.; CROMBLEHOLME, T. M.; LANGER, J. C.; VERRIER, E. D.; TURLEY, K. Systemic-to-pulmonary artery shunt using the internal mammary artery. Ann. Thorac. Surg., 47: 464-465, 1989.

18 MEARNS, A. J.; DEVERALL, P. B.; KESTER, R. C. Revascularization of an arm for incipient gangrene after Blalock-Taussig anastomosis. Br. J. Surg., 65: 467-468, 1978.

19 MOULTON, A. L.; BRENNER, J. I.; RINGEL, R.; NORDENBERG, A.; BERMAN, M. A.; ALI, S.; BURNS, J. Classic versus modified Blalock-Taussig shuns in neonates and infants. Circulation, 72 (Supl. 2): 35-44, 1985.

20 NORBERG, W. J.; TADAVARTHY, M.; KNIGHT, L.; NICOLOFF, D. M.; MOLLER, J. H. - Late hemodynamic and angiographic findings after ascending aorta-pulmonary artery anastomosis. J. Thorac. Cardiovasc. Surg., 76: 345-352, 1978.

21 POTTS, W. J.; SMITH, S.; GIBSON, S. - Anastomosis of the aorta to a pulmonary artery: certain types in congenital heart disease. JAMA, 132: 627-631,1946.

22 SIEVERS, H.; LANGE, P.; HEINTZEN, P. - Internal mammary artery as a palliative systemic-pulmonary shunt in order to develop diminutive pulmonary arteries. Thorac. Cardiovasc. Surgeron, 33: 51-61, 1984.

23 SKOVRÁNEK, J.; GOETZOVÁ, J.; SAMÁNEK, M. - Changes in muscle blood flow and development of the arm following the Blalock-Taussig anastonosis. Cardiology, 61: 131-137, 1975

24 SOMERVILLE, J.; YACOUB, M.; ROSS, D. N.; ROSS, $\mathrm{K}$. - Aorta-to-right pulmonary artery anastomosis (Waterston's operation) for cyanotic heart disease. Circulation, 39: 593-602, 1969.

25 STEWART, S.; HARRIS, P.; MANNING, J. - Current results with construction and interruption of the Waterston anastomosis. Ann. Thorac. Surg., 25: 431-437, 1978.

26 TRUCCONE, N. J.; BOWMAN Jr., F. O.; MALM, J. R.; GERSONY, W. M. - Systemic-pulmonary arterial shunts in the first year of life. Circulation, 49:508-511, 1974.

27 VETTER, V. L.; RASHKIND, W. J.; WALDHAUSEN, J. A. - Ascending aorta-right pulmonary artery anastomosis: long-term results in 137 patients with cyanotic congenital heart disease. J. Thorac. Cardiovasc. Surg., 76: 115-125, 1978.

28 WATERSTON, D. J. - The treatment of Fallot's tetralogy in infants under the age of one year. Rozhl. Chir. 41: $181-183,1962$.

29 WATERSTON, D. J.; STARK, J.; ASHCRAFT, K. W. Ascending aorta to right pulmonary artery shunts: experience with 100 patients. Surgery, 72:897-905, 1972. 
CARNEIRO, J. J.; VICENTE, W. V. A.; QUEIROZ, O. A.; TANNÚS FILHO, J. M.; MARIN NETO, J. A.; GRANZOTTI, J. A.; SADER, A. A. - Anastomose mamária interna-artéria pulmonar para o tratamento paliativo das cardiopatias congênitas cianóticas. Rev. Bras. Cir. Cardiovasc., 5(2): 79-85, 1990.

30 WILSON, J. M.; MACK, J. W.; TURLEY, K.; EBERT, P. A. - Persistent stenosis and deformity of the right pulmonary artery after correction of the Waterston anastomosis. J. Thorac. Cardiovasc. Surg., 82: 169-175, 1981.

\section{Discussāo}

DR. PAULO P. PAULISTA

São Paulo, SP

Nossos cumprimentos ao Dr. Joâo Carneiro e colaboradores, pela bela apresentação e, principalmente, pela escolha do tema, reavivando, em nossa memória, uma técnica cirúrgica interessante e pouco conhecida e na qual, com sua apresentaçāo, ele se torna o autor com a maior experiência. As vantagens que a literatura cita para o uso das mamárias na realidade são superponiveis para a subclávia na operação de Blalock-Taussig clássica, com excessão do sacrifício da circulação direta do membro superior correspondente. Influenciados pela leitura do trabalho do Dr. Carneiro, há dez dias fizemos uma anastomose mamária esquerda-artéria pulmonar esquerda em paciente com dois anos e cinco meses de idade portadora de atresia tricúspide com artéria esquerda não confluente e hipoplásica, só visivel no wedge venoso e que sobrevivia às custas da operação de Blalock-Taussig à direita feita no paciente com dois meses de idade. No ato cirúrgico, a artéria pulmonar esquerda tinha menos de $4 \mathrm{~mm}$ de diâmetro e a mamária utilizada, 2,0 $\mathrm{mm}$. A anastomose término-lateral foi feita em extensão de $5 \mathrm{~mm}$ na artéria pulmonar esquerda, semelhante à técnica da anastomose mamária-coronária e em posição que nos pareceu a mais adequada. Com base neste caso único, o que é muito pouco, restou-nos a impressão de que a técnica deve ser feita em pacientes que não serão imediatamente dependentes da mamária, fora, portanto, de emergências, que tenham artéria pulmonar hipoplásica e, se possível, artéria mamária calibrosa, visivel ao exame contrastado prévio, ou no ato cirúrgico. Muito obrigado.

\section{DR. CARLOS S. FIGUEROA \\ Belo Horizonte, MG}

A anastomose sistêmico-pulmonar é, se dúvida, amplamente empregada nas cardiopatias congênitas com hipofluxo pulmonar, em pacientes nos quais não é possivel a correçăo total. Também é verdade que a sua utilização tem diminuído devido à melhoria das condiçōes técnicas dos nossos centros de cirurgia cardiaca pediátrica, possibilitando a correção total das cardiopatias numa idade mais precoce. A grande maioria das técnicas para a realização da anastomose sistêmico-pulmonar já foram realizadas pela nossa equipe. Hoje, ficamos com a que achamos ser mais prática, segura e menos traumática para os pacientes. O seu emprego se baseia no princípio de que, sendo uma cirurgia em geral paliativa, a sua permeabilidade não é desejada para mais do que cinco anos, época na qual é feita a correção total. Usamos com enxerto na anastomose sistêmico-pulmonar o tubo de PTEF (politetrafluoroetileno) com diâmetro de $4 \mathrm{~mm}$ para neanatos e $5 \mathrm{~mm}$ para crianças maiores. No primeiro grupo a anastomose é na aorta torácica, junto à origem da artéria subclávia, e no segundo grupo, com esta artéria. Em relaçāo ao trabalho do Dr. Joāo José Carneiro e seu grupo, achei muito bem redigido e apresentado, com citação de vasta literatura, inclusive o trabalho original a respeito desta técnica. Gostaria, entretanto, que o Dr. Carneiro nos respondesse: 1) foram submetidos a correção total dois pacientes: um com 10 $\mathrm{kg}$ de peso, boa anatomia um mês após a cirurgia paliativa, sendo que o enxerto estava pérvio; se a anastomose estava funcionando, porque realizar a correção total tão precocemente? 2) achei a mortalidade elevada, dois pacientes faleceram no grupo de sete operados, o que dá uma percentagem de $28,5 \%$. É verdade que poderia ser evitada se fossem empregadas pinças hemostáticas mais delicadas. Para terminar, quero parabenizar o $\mathrm{Dr}$. Carneiro e seu grupo, pela originalidade do trabalho no nosso meio e desejar que, em futuro Congresso, possa nos falar, com maiores dados, da utilidade do método. Obrigado.

\section{DR. CARNEIRO \\ (Encerrando)}

Nossos agradecimentos aos Drs. Figueroa e Paulo Paulista, por seus comentários. Quanto à paciente de $10 \mathrm{~kg}$, portadora de Fallot com boa anatomia, foi submetida a anastomose mamária interna-artéria pulmonar, devido a suas precárias condições gerais (saindo de pneumonia de repetição e apresentando crises hipoxêmicas freqüentes, apesar do tratamento clinico). Sua melhora, ao final de um mês, foi tão acentuada que, em função de suas condiçōes sócio-econômicas e culturais, reconsideramos o caso e a submetemos a correção total, com sucesso. A mortalidade em nossa série é alta $(28,5 \%)$ e julgamos estar relacionada à gravidade dos pacientes operados por técnica mais delicada. Para estes casos, seguimos preferindo anastomoses mais rápidas e de mais fácil execução, como o da técnica de Blalock-Taussig modificada. Concordamos, pois, com os critérios de indicação colocados pelo Dr. Paulo Paulista, em seus comentários. Omitimos o calibre das mamárias e pulmonares, por dispormos apenas de parte desses dados. Consideramos a anastomose mamária interna-artéria pulmonar uma boa opção a mais para as derivaçōes sistêmico-pulmonares e não simplesmente mais uma técnica dentre as muitas já existentes. Estamos convictos de sua indicação e de bons resultados em casos especialmente selecionados. Obrigado. 